\title{
Authorship Correction: A Clinician-Controlled Just-in-time Adaptive Intervention System (CBT+) Designed to Promote Acquisition and Utilization of Cognitive Behavioral Therapy Skills in Bulimia Nervosa: Development and Preliminary Evaluation Study
}

Adrienne Juarascio, PhD; Paakhi Srivastava, PhD; Emily Presseller, BA; Kelsey Clark, MA; Stephanie Manasse, $\mathrm{PhD}$; Evan Forman, PhD

Center for Weight, Eating and Lifestyle Science, Drexel University, Philadelphia, PA, United States

\section{Corresponding Author:}

Paakhi Srivastava, $\mathrm{PhD}$

Center for Weight, Eating and Lifestyle Science

Drexel University

3141 Chestnut Street, Stratton Hall, Suite 232

Philadelphia, PA, 19104

United States

Phone: 12678156511

Email:ps887@drexel.edu

\author{
Related Article: \\ Correction of: https://formative.jmir.org/2021/5/e18261
}

(JMIR Form Res 2021;5(7):e31964) doi: 10.2196/31964

In "A Clinician-Controlled Just-in-time Adaptive Intervention System (CBT+) Designed to Promote Acquisition and Utilization of Cognitive Behavioral Therapy Skills in Bulimia Nervosa: Development and Preliminary Evaluation Study" (JMIR Form Res 2021;5(5):e18261), one error was noted.

In the originally published article, the authorship order was incorrect. The article was originally published with the following authorship order:

Adrienne Juarascio, Paakhi Srivastava, Kelsey Clark,

Emily Presseller, Stephanie Manasse, Evan Forman
In the corrected article, the authorship order has been updated as follows:

\section{Adrienne Juarascio, Paakhi Srivastava, Emily \\ Presseller, Kelsey Clark, Stephanie Manasse, Evan Forman}

The correction will appear in the online version of the paper on the JMIR Publications website on July 29, 2021, together with the publication of this correction notice. Because this was made after submission to PubMed, PubMed Central, and other full-text repositories, the corrected article has also been resubmitted to those repositories.

This is a non-peer-reviewed article. Submitted 13.07.21; accepted 15.07.21; published 29.07.21.

Please cite as:

Juarascio A, Srivastava P, Presseller E, Clark K, Manasse S, Forman E

Authorship Correction: A Clinician-Controlled Just-in-time Adaptive Intervention System (CBT+) Designed to Promote Acquisition and Utilization of Cognitive Behavioral Therapy Skills in Bulimia Nervosa: Development and Preliminary Evaluation Study

JMIR Form Res 2021;5(7):e31964

URL: https://formative.jmir.org/2021/7/e31964

doi: $\underline{10.2196 / 31964}$

PMID: 34324426

(C)Adrienne Juarascio, Paakhi Srivastava, Emily Presseller, Kelsey Clark, Stephanie Manasse, Evan Forman. Originally published in JMIR Formative Research (https://formative.jmir.org), 29.07.2021. This is an open-access article distributed under the terms of the Creative Commons Attribution License (https://creativecommons.org/licenses/by/4.0/), which permits unrestricted use, distribution, and reproduction in any medium, provided the original work, first published in JMIR Formative Research, is properly 
cited. The complete bibliographic information, a link to the original publication on https://formative.jmir.org, as well as this copyright and license information must be included. 